\title{
INR ACTIVITY IN DEVELOPMENT AND PRODUCTION OF BUNCH SHAPE MONITORS
}

\author{
S.K.Esin, A.V.Feschenko, P.N.Ostroumov, Institute for Nuclear Research, 117312 Moscow, Russia
}

Precise measurements of the longitudinal profiles of ion beams with $\sim 10 \mathrm{ps}$ temporal resolution became available with the help of a Bunch Shape Monitor (BSM) developed and used at the INR. A number of BSMs have been developed, fabricated at the INR and tested with a beam at several laboratories during last 3 years. The upgraded BSM-Bunch Length and Velocity Detector (BLVD)-can measure not only longitudinal profile but also absolute beam velocity. A 3D$\mathrm{BSM}$ is a further improvement of the monitor which allows a three-dimensional density distribution of the bunched beam to be measured.

\section{INTRODUCTION}

The BSMs are normally used to measure a bunch longitudinal density distribution at present. In these monitors the temporal structure of the beam under study is coherently transformed into that of low energy secondary electrons and then into a spatial structure by RF modulation. The first proposal to use low energy secondary electrons for this purpose was made in the early sixties [1]. To obtain a phase dependent separation of the electrons it was suggested that their velocity be modulated transversally in two perpendicular directions by a circular scan. The first real device was proposed and created in the mid seventies [2]. In this device an RF field was used to obtain longitudinal or energy modulation of the electrons. Phase separation of the electrons was obtained using a magnetic field.

In order to improve the phase resolution, a monitor with transverse modulation of the velocity of the electrons has been developed and built at the INR. In this monitor, the velocity of the electrons is transversally modulated by an RF sweep field in one direction providing a phase dependent separation after a drift space [3]. This device has been installed in the INR linac and is being used successfully to tune the accelerator and to test the beam quality. Monitors of this type have found application in a number of accelerators. Two devices have been developed and designed with our assistance at Fermilab [4]. Recently, four monitors have been built at INR for the SSC linac [5,6]. We have just completed development and design and have began fabrication of two BSMs for the DESY proton linac.

The devices mentioned above can be considered as traditional ones. A number of ideas have been proposed recently for the improvement of the traditional monitor. The BLVD provides a measurement of the average beam velocity as well as the bunch shape [7]. This detector has been developed, designed and built at the INR for the CERN Heavy Ion Linac [8]. Detectors of this type have been developed and are being fabricated for the DESY proton linac as well as for the INR linac. The traditional BSM can be modernized to measure longitudinal and transverse distributions of beam bunches including two component beams [9]. A further improvement of the monitor is a 3D-BSM - a detector, which allows the measurement of the charge density distribution in a 3-dimensional space [10]. This monitor is being fabricated now at INR for the CERN proton linac.

\section{BUNCH SHAPE MONITORS}

The arrangement of the BSM with transverse modulation of low energy secondary electrons is reported elsewhere [3,11]. A BSM consists of the following main elements: a target, an input collimator, an electrostatic lens, an RF deflector, an output collimator and, following a drift space, an electron current detector.

Table 1

\begin{tabular}{|l|l|l|}
\hline Deflector type & $\mathrm{f}, \mathrm{MHz}$ & Lab \\
\hline two coupled $\lambda / 2$ coaxial & 594.6 & INR \\
\hline two coupled $\lambda / 2$ coaxial & 202.56 & DESY \\
\hline$\lambda / 4$ parallel wire line & 427.6 & SSCL \\
\hline$\lambda / 4$ parallel wire line & 202.56 & CERN \\
\hline $5 \lambda / 4$ parallel wire line & 1282.8 & SSCL \\
\hline$\lambda / 2$ parallel wire line & 991 & INR \\
\hline$\lambda / 2$ parallel wire line & 810.24 & DESY \\
\hline
\end{tabular}

- The general configuration of all of the BSMs we have developed is practically the same but specific features and designs differ and are determined by mechanical and electrical requirements as well as by beam parameters. The main component of a BSM is the RF deflector.

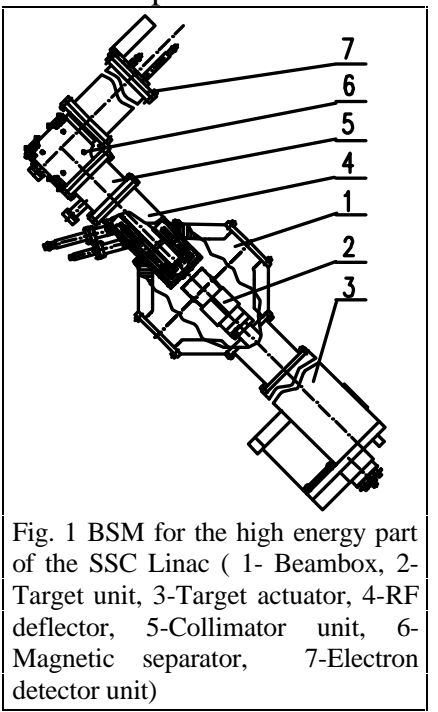

Since 1992 we have used an RF deflector combined with electrostatic lenses in a single unit [9]. This combination allows improvement of the monitor parameters and avoids multipactor discharge due to the HV focusing potential applied to the deflecting plates. A number of RF deflectors have been developed (see Table 1). All of the deflectors consume $10 \mathrm{~W}$ RF power to provide a nominal value of deflecting field. Fig. 1 shows one of the four BSMs built for the SSC

Laboratory. The devices fit into the standard beam box with a dimension along the beam line $80 \mathrm{~mm}$. The monitor shown was intended to be used in the high energy section of the 
accelerator. It utilizes an additional magnetic separator to prevent the influence of the electrons stripped from $\mathrm{H}^{-}$ions. The DESY BSMs have been designed to be installed into the ports of the existing intertank sections.

The main parameter of the BSM is its phase resolution which depends upon a number of factors. A resolution of 1 degree for frequencies up to $400 \mathrm{MHz}$ can be achieved.

\section{BUNCH LENGTH AND VELOCITY DETECTORS}

Time of flight methods have been used to measure the average velocity of beams with an RF bunch structure. Either two monitors installed a known distance apart [12] or a single movable one [13] can be used for this purpose. A BSM can be used as a movable detector [7]. When moving the BSM in a longitudinal direction one can observe a change in the bunch phase shape location by a value $\Delta \varphi_{0}=2 \pi d / \beta \lambda$, where $\beta$ is the relativastic velocity of the beam, $\lambda$ is the wavelength of the deflector RF field and $d$ is the distance of the monitor displacement. By measuring $\Delta \varphi_{0}$ and $d$ one can find beam velocity. An improved monitor capable of measuring the average velocity, the BLVD, has been developed and built at INR for the CERN Heavy Ion Linac [8]. A general view of the BLVD is shown in fig. 2. The detector was used for $\mathrm{Pb}^{+27}$ ions with $\beta=0.02$ and $\beta=0.09$ and provided energy

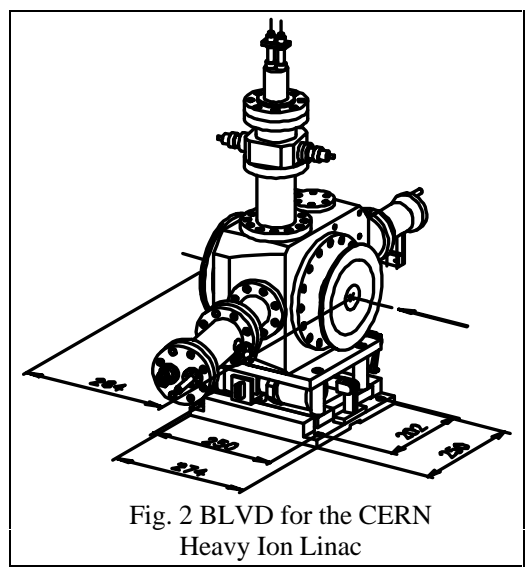
measurements with an accuracy of about $0.15 \%$ and $0.3 \%$ respectively.

The best accuracy is obtained when the value of the detector translation is exactly equal to $\beta \lambda$. If the translation equals $\beta \lambda / 2$ the accuracy suffers a slight degradation but only half as much mechanical translation is required. For a high $\beta$ it is expedient to use a higher harmonic for the deflecting field. The BLVD for the DESY Linac, for which the design has recently been completed, will operate at the fourth harmonic, $f=810.24$ $\mathrm{MHz}$. For the $50 \mathrm{MeV}$ protons the value of the detector translation, $\beta \lambda / 2$, is $60 \mathrm{~mm}$.

\section{DETECTORS TO MEASURE THREE DIMENSIONAL DENSITY DISTRIBUTION}

The name of the device, Bunch Shape Monitor, is not exactly correct. In the main, it is intended for the measurement of the longitudinal distribution of only those particles which impinge on the wire but not of a real bunch shape which is a function of the three coordinates.
To measure a three dimensional charge density distribution one can use the fact that the target is thin with respect to its distance from the deflector. A schematic diagram of the $3 \mathrm{D}-\mathrm{BSM}$ is shown in fig. 3. The target is surrounded by a grounded shield. The target, shield, and collimator are moved in the $y$-direction simultaneously. In addition, the collimator can be moved independently in the $x$ direction with the help of the pusher. The width of the collimator, along the target, is much smaller than the beam transverse dimension. Thus the intensity of the electrons, passed through the collimator, is proportional to the intensity of the primary ions at a definite point of the transverse $(x, y)$ plane. By moving the collimator horizontally, one can get information about the beam for different $x$-coordinates. Information for other $y$-coordinates can be obtained by moving the target, shield, and collimator vertically. By measuring the longitudinal distribution for each vertical location of the target and each horizontal location of the collimator one can obtain a 3-coordinate charge density distribution function. Spatial resolution in the $x$-direction depends upon a number of parameters and was estimated to be about $1 \mathrm{~mm}$. To decrease the total duration of the measurements, the longitudinal distribution is measured within one beam pulse with the use of a multichannel collector.

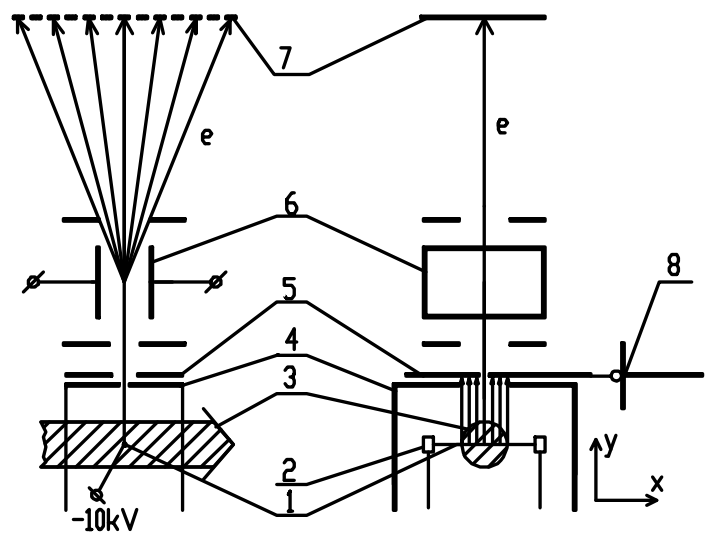

Fig. 3 Schematic diagram of the 3D-BSM (1-target, 2-target holders, 3-beam under study, 4-shield, 5-movable collimator, 6-RF deflector, 7-multichannel collector, 8-pusher)

The variable distance between the input collimator and the RF deflector entrance is transversed by the electrons with a constant and well known velocity thus enabling a simple restoration of the relative phase locations of longitudinal distributions measured for different $y$-coordinates. A slight variation of phase resolution due to changed focusing of the electrons for different target locations is a relatively small deficiency of the configuration. This configuration was selected for the 3D-BSM which we are now developing for the CERN Linac 2.

\section{APPLICATION OF GAS TARGET}


To measure high intensity ion beam parameters a gas target can be used as a source of secondary electrons. The molecular gas jet must have small transverse dimensions. A uniform electrostatic field, $\sim 10 \mathrm{kV} / \mathrm{cm}$, must be applied to

Table 2

\begin{tabular}{|c|c|c|}
\hline Proton energy & $100 \mathrm{MeV}$ & detached \\
\hline Peak current & $10 \mathrm{~mA}$ & The contribution \\
\hline Pulse length & $100 \mu \mathrm{s}$ & the finite jet size \\
\hline Beam diameter & $1 \mathrm{~cm}$ & the electron time-c \\
\hline Gas jet size & $1 \mathrm{~mm}$ & flight and ener \\
\hline $\begin{array}{l}\text { Distance from the jet to } \\
\text { the zero potential plane }\end{array}$ & $2 \mathrm{~cm}$ & $\begin{array}{l}\text { spread can } \\
\text { compensated by usin }\end{array}$ \\
\hline $\begin{array}{l}\text { Magnetic spectrometer } \\
\text { resolution } \Delta p / p\end{array}$ & $10^{-2}$ & a mag \\
\hline $\begin{array}{l}\text { Concentration of air } \\
\text { molecules in the target }\end{array}$ & $10^{13} \mathrm{~cm}^{-3}$ & $\begin{array}{l}\text { to the one shown } \\
\text { fig. } 1 . \text { For }\end{array}$ \\
\hline
\end{tabular}

parameters listed in Table 2 the number of electrons at the entrance of the electron collector is $\sim 10^{4}$ when the RF deflector is off. For a continuous gas flux and a $10^{7}$ torr pressure a $1400 \mathrm{l} / \mathrm{s}$ pumping speed is required. This value can be reduced to a reasonable one if a pulsed bleed-in is used.

\section{SENSITIVITY OF BSM}

The sensitivity of the BSM becomes of importance during the measurement of a low intensity beam or a longitudinal halo of a relatively high intensity beam.

Normally electron multipliers are used in BSMs to detect secondary electrons. Typical values of the maximum gain of the multipliers for different types of the devices are $\sim 10^{5}-10^{8}$ and can be adjusted over three to four orders of magnitude by varying the HV. The multipliers can detect individual electrons so, as a matter of principle, by increasing the duration of the measurements it is possible to measure longitudinal parameters of extremely low intensity beams. Practically, the sensitivity is limited in any specific case. Our experience allows us to distinguish the following cases:

1. Detection of the low intensity longitudinal halo of a relatively high intensity (about $10 \mathrm{~mA}$ ) beam. In this case the sensitivity is limited by radiation background because of beam losses including inevitable losses due to interaction with the target. Regular measurements at the exit of the low energy section $(100 \mathrm{MeV})$ of the INR linac have shown that it is relatively easy to detect longitudinal halo on the relative level of $10^{-3}$. To detect halo on the level of $10^{-4}$ a special measurement procedure must be performed. The effect under consideration depends upon a number of factors: beam energy, type of particles, beam dimensions, etc.

2. Detection of longitudinal parameters of a low intensity beam. The minimum beam peak current we have made measurements with at the exit of the low energy section of the INR linac was abut $3 \mu \mathrm{A}$. In this case, the statistical nature of the signal is observed and amplitude resolution deteriorates due to the relatively small number of detected electrons. Integration of the signal within a beam pulse $(80 \mu \mathrm{s})$ provides an acceptable amplitude resolution. A further decrease of intensity will result in the necessity to detect individual electrons. Measurements at the CERN Heavy Ion Linac have been made for the $0.25 \mathrm{MeV} / \mathrm{amu}$ (exit of the RFQ) and 4.2 $\mathrm{MeV} / \mathrm{amu}$ (exit of the accelerator) $\mathrm{Pb}^{+27} 10 \mu \mathrm{A}$ beams [8]. At the exit of the accelerator a background signal originated from positive ions impinging on the negative potential target which was synchronous with the RF in the IH accelerating tanks. Due to this effect, the beam current to be analyzed has been limited to $1 \mu \mathrm{A}$.

\section{CONCLUSION}

A number of BSMs and modified detectors have been developed and built or are being built at INR for several laboratories. The devices have extremely high resolution and sensitivity. Due to differing beam parameters, frequencies, and mechanical and electrical requirements an individual approach to the research, development, and design of each monitor is necessary. Modern detectors like the BLVD can be used in place of conventional bulky and expensive diagnostic installations yet provide information otherwise inaccessible, e.g. by a 3D-BSM.

\section{REFERENCES}

[1] I.A.Prudnikov et all. A Device to Measure a Bunch Phase Length of Accelerated Beam. USSR invention license, H05h7/00, No.174281 (in Russian).

[2] R.L.Witkover. A Non-Destructive Bunch Length Monitor For a Proton Linear Accelerator, NIM, 137 (1976), pp. 203-211.

[3] A.V.Feschenko and P.N.Ostroumov. Bunch Shape Monitor and Its Application for an Ion Linac Tuning. Proc. of the 1986 Linac Conf., Stanford, June 2-6, pp. 323-327.

[4] E.S.McCrory, C.W.Schmidt and Use of an INR-Style Bunch Length Detector in the Fermilab Linac. Proc. of the Linac-92, Ottawa, August 24-28, 1992, pp. 662-664.

[5] J.M.Hurd et all. Bunch Shape Monitor for SSCL Linac. Proc. of the 1993 PAC, Washington, 1993, pp. 2426-2428.

[6] S.K.Esin et all. Development and Fabrication of Bunch Shape Monitors For SSCL Linac. Reported at the 14th All Russia Meeting on Particle Accelerators. Protvino. October 25-27, 1994. Be published. (In Russian)

[7] P.N.Ostroumov. Average Velocity Measurement of Accelerated Beam by Means of an Upgraded Bunch Length Detector. INR Preprint-812/92, May 1993.

[8] Yu.V.Bylinsky et all. Bunch Length and Velocity Detector and Its Application in the CERN Heavy Ion Linac. Proc. of the EPAC-94, London, June 27-July 1,1994.

[9] A.V.Feschenko, A.A.Men'shov, P.N.Ostroumov. A Detector to Measure Longitudinal and Transverse Distributions of a Two Component Ion Beam. Proc. of the EPAC-92, Berlin, 24-28 March, 1992, V.2, pp. 1073-1075

[10] S.K.Esin, A.V.Feschenko. Detector of Three Dimensional Charge Distribution in Linear Ion Accelerator. Reported at the 14th All Russia Meeting on Particle Accelerators. Protvino. October 25-27, 1994. Be published. (In Russian)

[11] A.V.Feschenko. Bunch Shape Monitors Using Low Energy Secondary Electron Emission. AIP Conf. Proc. No. 281, Particles and Fields, Series 52, Accelerator Instrumentation Forth Annual Workshop, Berkeley, Ca 1992, p.185-193

[12] Linear Ion Accelerators. Ed. by B.P.Murin, V.2, Atomizdat, 1978 (in Russian)

[13] A.V.Feschenko et all. Precise Energy Measurement of the Continuous Proton Beam. Proc. of the 1989 IEEE Particle Acc. Conf., Chicago, 1989, V.2. 